\title{
Etude des déficiences de la fertilité de l'épi chez différentes lignées de blé tendre et leurs hybrides en liaison avec leur comportement méiotique
}

\author{
Gérard DOUSSINAULT, Françoise DOSBA*, Françoise ROUSSELLE** \& Gérard DOUAIRE*** \\ avec la collaboration technique de Anne-Marie TANGUY* \\ I.N.R.A, Station d'Amélioration des Plantes, Centre de Recherches de Rennes, Domaine de la Motte-au- \\ Vicomte, B.P. 29, F 35650 Le Rheu \\ * I.N.R.A., Station de Recherches d'Arboriculture fruitière, Centre de Recherches de Bordeaux, La Grande \\ Ferrade, F 33140 Pont-de-la-Maye \\ ** I.N.R.A., Station d'Amélioration de la Pomme de terre et des Plantes à Bulbes, Centre de Recherches de \\ Rennes, Domaine de Kéraiber, Ploudaniel, F 29260 Lesneven \\ *** E.N.S.A.R., Chaire de Mathématiques Appliquées, 65, rue de Saint-Brieuc, F 35042 Rennes Cedex
}

RÉSUMÉ

Dans certaines conditions de températures basses peu avant la méiose, on observe une réduction de la production de pollen et de la nouaison chez le blé. Ces phénomènes sont particulièrement manifestes chez les lignées demi-naines issues de Norin 10, les descendants du géniteur VPM issus d'hybridations interspécifiques et surtout chez les hybrides entre ces 2 groupes. Nous avons fait l'hypothèse que des irrégularités méiotiques pouvaient expliquer cette situation.

Une analyse du comportement méiotique, de la fertilité pollinique et de la nouaison a été réalisée sur 4 lignées : " Maris Freeman » (T), "(Champlein X Aronde) 68 " (C), «VPM 1.1.1.2.R4 » (V) et " Naünari 60S » (U); sur les hybrides $F 1$ entre ces lignées, sur certains rétrocroisements et sur des plantes F2 du croisement (VU). Nous avons défini un critère de mesure du déficit de fertilité de l'épi, le taux de nouaison, qui est la probabilité d'obtenir un grain dans les 2 premières fleurs de l'épillet. L'observation des 4 premiers épillets fertiles de la base de l'épi semble être suffisante pour réaliser cette estimation.

Une corrélation nette a pu être mise en évidence entre le pourcentage de grains de pollen anormaux et le taux de nouaison.

Certains génotypes ont des taux de nouaison faibles comme $T$ et surtout $V$. Ils produisent des $F 1$ dont le taux de nouaison dépend du partenaire avec lequel ils sont combinés.

Nous observons un taux de nouaison plus faible en moyenne en F2 qu'en F1. L'effet du cytoplasme semble faible.

Le comportement méiotique des hybrides est moins régulier que celui des parents. Un excès d'univalents et de multivalents traduit qu'l ou 2 translocations réciproques importantes différencient les parents mis en présence. Les irrégularités méiotiques importantes induisent toujours une baisse du taux de nouaison, cependant une méiose régulière ne conduit pas obligatoirement à un taux de nouaison élevé. Les défauts d'association des chromosomes en métaphase I n'expliquent donc que très partiellement la réduction de nouaison observée.

Mots clés additionnels : Interaction génotype milieu, climat, stérilité mâle, appariement chromosomique, translocation chromosomique.

After a cold spring, a decrease in pollen production and seed set was observed in wheat. These phenomena were most marked with semi-dwarf lines derived from Norin 10, progenies of interspecific crosses and particularly among the hybrids between these two groups. Irregular meiotic behaviour could possibly explain this situation. Meiotic behaviour, pollen fertility and seed set were analysed on 4 lines: "Maris Freeman" (T), "(Champlein $\times$ Aronde) 68" (C), "VPM 1.1.1.2.R4" (V) and "Nainari 60S" (U), on the F1 hybrids between these lines, some back-cross plants and F2 plants from the (VU) cross. Lack of fertility was defined as the level of seed set corresponding to a probability of one grain in the first 2 florets of the spikelet. Observation of the first 4 spikelets at the base of the spike gave a good estimate of the level of fertility. A correlation was found between the percentage of abnormal pollen and seed set level. Some genotypes showed a seed set level as low as $\mathrm{T}$ and most as low as V. The seed set of their F1 depended on the other parent. On average, the seed set level was lower in F2 than in F1. The cytoplasmic effect seemed to be low. The meiotic behaviour of the hybrids was less regular than that of the parents. Excess of univalents and multivalents showed that one or two reciprocal translocations existed between the parents. Irregular meiosis always induced a decrease in seed set level ; nevertheless regular meiosis did not always indicate a high seed set level. Lack of chromosome pairing at metaphase I was not sufficent to explain the decrease of seed set.

Additional key words : Genotype, environment interaction, climate, male sterility, chromösome pairing, chromosome transiocation. 


\section{INTRODUCTION}

Chez le blé tendre: Triticum aestivum (L.) Thell ssp. vulgare M.K., nous avons observé une stérilité importante, due à un défaut de nouaison, en 1973, après un printemps anormalement froid : 3 journées consécutives avec des températures minimales inférieures à $3{ }^{\circ} \mathrm{C}$ et des températures moyennes inférieures à $10{ }^{\circ} \mathrm{C}$ dans la semaine précédant la méiose. Cette déficience de nouaison a été particulièrement marquée chez des lignées demi-naines d'origine américaine possédant les gènes de nanisme de "Norin 10 », chez des lignées sélectionnées en France, issues d'hybridations interspécifiques faisant intervenir Aegilops ventricosa Tausch et surtout chez des hybrides F1 entre ces 2 groupes de lignées.

Cet accident s'est reproduit sur des variétés cultivées en France ou dans l'Europe du Nord Ouest comme «Pernel » en 1984 dans des circonstances climatiques similaires. Il constitue un problème de plus en plus fréquent parmi les variétés de blé. Les mécanismes intervenant dans ce phénomène peuvent bien entendu être différents selon les génotypes.

Déjà SUNESON (1962) a signalé que certaines variétés présentent des stérilités favorisées par les températures basses avant l'épiaison. Chez la tomate, B. MAISONNEUVE \& J. Philouze (1982), observent que des températures basses durant la floraison diminuent la quantité et la qualité du pollen de façon variable selon les génotypes. En 1978, BANNIER trouve une relation entre la fertilité et le comportement méiotique chez le blé. " Norin 10 », qui exprime particulièrement ce caractère de stérilité, présente par ailleurs des irrégularités méiotiques avec présence d'univalents à la méiose (WATANABE, 1954); mais aucune relation entre ces 2 observations n'a été établie.

BENNETT et al. (1972) ayant démontré l'incidence de la température sur les appariements chromosomiques, nous avons émis l'hypothèse que la stérilité observée dans nos conditions pouvait être due à des irrégularités méiotiques.

Nous nous sommes proposés d'étudier cette stérilité à l'aide de croisements faisant intervenir des géniteurs ayant donné des hybrides stériles en 1973. Des critères de mesure de stérilité des épis ont été établis ; une comparaison de la stérilité et de la régularité de la méiose a été réalisée aux générations $F 1$ et $F 2$.

\section{MATÉRIEL ET MÉTHODES}

\section{A. Matériel}

Quatre lignées ont été utilisées. Les 2 premières, la variété anglaise «Maris Freeman»(T) et la lignée obtenue à la Station d'Amélioration des Plantes de Rennes « (Champlein $\times$ Aronde) 68 » (C) ne possèdent pas de gène de nanisme et proviennent d'hybridations intraspécifiques. La lignée « V.P.M.1.1.1.2 R4 » (V) est un géniteur issu d'Aegilops ventricosa (MAIA, 1967). La variété mexicaine « Nainari $60 \mathrm{~S}$ » (U) porte le gène de nanisme Rht2 issu de « Norin 10 » (GALE et al., 1981).
Nous avons étudié les générations $F 1$ et $F 2$ des croisements réciproques entre $U$ et les 3 autres lignées ainsi que les $\mathrm{F} 1$ et $\mathrm{F} 2 \mathrm{CV}$ et $\mathrm{CT}$. Les rétrocroisements des F1 UV, VU et CV par les 2 lignées parentales ont aussi été analysés.

\section{B. Méthodes}

\section{Conditions expérimentales}

Le taux de nouaison en 1975 sur les parents et les F1 ainsi qu'en 1976 sur les parents, F1, F2 et rétrocroisements a été observé sur des plantes cultivées en chassis froids, à faible densité : 40 plantes au $\mathrm{m}^{2}$. Le comportement méiotique et la fertilité pollinique ont été observés sur les mêmes plantes, excepté en 1975 dans le cas des parents et les $\mathrm{F} 1$ dont le comportement méiotique a été réalisé sur des plantes cultivées en serre froide où les températures minimales n'ont jamais descendu audessous de $5{ }^{\circ} \mathrm{C}$.

En 1975, les températures minimales subies par les plantes varient de $2^{\circ} \mathrm{C}$ à $10^{\circ} \mathrm{C}$ durant les stades préméiotiques et méiotiques. Deux journées non consécutives, les 12 et 15 mai, ont présenté des températures faibles mesurées à $2 \mathrm{~m}$ sous abri aussi bien en ce qui concerne les minimales $\left(2,0^{\circ} \mathrm{C}\right.$ et $\left.3,8^{\circ} \mathrm{C}\right)$ que les moyennes journalières $\left(8,3^{\circ} \mathrm{C}\right.$ et $\left.7,9^{\circ} \mathrm{C}\right)$.

En 1976, la situation est différente ; la température minimale la plus basse au cours de la période couvrant les stades préméiotiques et méiotiques est de $5,0^{\circ} \mathrm{C}$ et la température moyenne journalière la plus basse est de $8,6^{\circ} \mathrm{C}$.

\section{Mesures de fertilité des épis}

Le nombre total de grains formés dans chaque épillet fertile est une caractéristique génotypique fortement influencée par les conditions de milieu. Dans nos essais, les facteurs de croissance n'ont pas été limitants et pour tous les génotypes, les 2 premières fleurs au moins ont toujours été potentiellement fertiles. Nous définissons le taux de nouaison comme le rapport du nombre de grains formés au nombre de grains potentiels dans les 2 premières fleurs de chaque épillet. Donc un déficit de nouaison serait dû à un mauvais fonctionnement des gamètes ou à un avortement du grain. Pour étudier ce phénomène nous avons dénombré les grains formés sur les 2 premières fleurs de chaque épillet. Afin d'éviter les allofécondations, les épis ont été isolés avant floraison par des sachets de cellophane. L'un des épis maîtres de chaque plante a été observé. Les analyses ont porté sur environ 15 plantes pour les parents, 10 à 30 plantes pour les $\mathrm{F} 1$ et les rétrocroisements et sur 40 à 60 plantes pour les $F 2$.

\section{Etudes cytologiques}

Les dénombrements des chromosomes en mitose et les observations méiotiques en métaphase réductionnelle (MI) ont été réalisés selon les techniques précédemment décrites (DosBa \& DoussinaulT, 1978). De 16 à 26 plantes des parents, de 3 à 8 plantes des $\mathrm{F} 1$ et 7 à 9 plantes des F2 UV et VU ont été observées. L'analyse du comportement méiotique en MI indique les défauts d'appariement des chromosomes par la présence d'univalents qui traduisent des phénomènes 
d'asynapsis ou de désynapsis. Elle révèle également, par le nombre et la fréquence de multivalents observés, certaines différences de structure chromosomique entre les parents de l'hybride concerné (RILEY et al., 1967 ; BOURGEOIS et al., 1978).

\section{Estimation de la fertilité pollinique}

La fertilité pollinique a été estimée à partir de grains de pollen prélevés à l'épiaison et à l'anthèse, colorés dans le carmin acétique de Belling. Les grains de pollen dégénérés se distinguent par leur forme anormale en plus d'un manque plus ou moins complet de coloration dû à l'absence de réserves d'amidon et de noyaux. Par contre, les grains colorés ne sont pas tous capables de féconder les ovules. Aussi la fertilité pollinique est surestimée par cette technique. Elle a été mesurée à l'épiaison et à l'anthèse sur 30 plantes F1 ou F2.

\section{RÉSULTATS ET DISCUSSION}

\section{A. Taux de nouaison}

\section{Etude des lignées parentales}

En moyenne, chez les lignées parentales, le taux de nouaison est relativement faible : 0,65 en 1975 et 0,74 en 1976.

On constate de plus des comportements différents dans les épillets de la base et ceux du sommet de l'épi.
Les dénombrements de grains dans les épillets de la base sont tels qu'il est possible d'admettre que chacune des 2 premières fleurs d'un même épillet produit un grain de manière indépendante de l'autre fleur. Par contre, il n'en est pas de même au sommet. Aussi, le nombre de grains produits dans les épillets de la base semble constituer un meilleur critère de mesure du taux de nouaison que celui des grains produits au sommet où se superposent probablement plusieurs phénomènes.

L'étude de la répartition des grains dans les différents épillets (tabl. 1) montre que pour toutes les lignées étudiées, le sommet est la partie la plus stérile de l'épi. Le taux de nouaison n'atteint pas encore la moyenne de l'épi au niveau du $4^{\mathrm{e}}$ épillet.

Le premier épillet de la base est légèrement moins fertile que la moyenne de l'épi, les suivants ont une fertilité analogue aux épillets médians.

Le comportement des lignées parentales n'est pas identique, la lignée $\mathrm{V}$, issue de croisement interspécifique, est globalement moins fertile que les autres (tabl. 2). En 1975, année la plus froide, le taux de nouaison sur l'ensemble de l'épi est moins bon qu'en 1976.

On observe une variabilité entre épis des différentes plantes. Pour l'épi entier et les épillets de la base, les valeurs des écarts-types varient de 15 à $40 \%$ de la moyenne, au niveau des épillets du sommet les écartstypes dépassent souvent $50 \%$ pour le même échantillon d'épi. Cette dernière observation confirme la difficulté d'utiliser le sommet de l'épi pour apprécier le taux de nouaison.

TABLEAU 1

Taux de nouaison au cours des 2 années d'observation; moyenne des 4 lignées parentales.

Seed set over two years; average of four parental lines.

\begin{tabular}{|c|c|c|c|c|c|}
\hline \multirow{2}{*}{$\begin{array}{l}\text { Numéro d'ordre } \\
\text { de l'épillet } \\
\text { à partir du sommet }\end{array}$} & \multicolumn{2}{|c|}{ Année d'observation } & \multirow{2}{*}{$\begin{array}{l}\text { Numéro d'ordre } \\
\text { de l'épillet } \\
\text { à partir de la base }\end{array}$} & \multicolumn{2}{|c|}{ Année d'observation } \\
\hline & 1975 & 1976 & & 1975 & 1976 \\
\hline 1 & 0,27 & 0,27 & 1 & 0,65 & 0,57 \\
\hline 2 & 0,37 & 0,45 & 2 & 0,72 & 0,71 \\
\hline 3 & 0,39 & 0,59 & 3 & 0,80 & 0,81 \\
\hline 4 & 0,58 & 0,62 & 4 & 0,82 & 0,82 \\
\hline Moyenne du sommet & 0,40 & 0,48 & Moyenne de la base & 0,75 & 0,73 \\
\hline Moyenne de l'épi entier & 0,65 & 0,74 & Moyenne de l'épi entier & 0,65 & 0,74 \\
\hline
\end{tabular}

TABLEAU 2

Taux de nouaison des 4 lignées parentales selon les secteurs de l'épi au cours des 2 années. Seed set in four parental lines according to their place in the spike over two years.

\begin{tabular}{|c|c|c|c|c|c|c|}
\hline & \multicolumn{2}{|c|}{ Dans tous les épillets de l'épi } & \multicolumn{2}{|c|}{ Dans les 4 épillets du sommet } & \multicolumn{2}{|c|}{ Dans les 4 épillets de la base } \\
\hline Lignées & 1975 & 1976 & 1975 & 1976 & 1975 & 1976 \\
\hline $\mathrm{T}$ Maris Freeman & 0,60 & 0,79 & 0,35 & 0,42 & 0,68 & 0,82 \\
\hline V VPM & 0,47 & 0,60 & 0,26 & 0,22 & 0,61 & 0,68 \\
\hline C (Champlein $\times$ Aronde) 68 & 0,73 & 0,76 & 0,54 & 0,64 & 0,77 & 0,66 \\
\hline Moyenne des lignées & 0,65 & 0,74 & 0,40 & 0,48 & 0,75 & 0,73 \\
\hline
\end{tabular}




\section{Etude des F1, F2 et rétrocroisements}

On observe un taux de nouaison moyen plus faible en F2 qu'en F1 aussi bien pour les 4 épillets de la base que pour l'épi entier (tabl. 3).

Le comportement en F1 paraît être un bon indicateur de ce que l'on observera en F2, par exemple les croisements UV et VU ont le taux de nouaison le plus faible aussi bien en F1 qu'en F2 pour l'épi entier.

Par ailleurs, les écarts-types sont systématiquement plus importants en $\mathrm{F} 2$ ou en rétrocroisement qu'en $\mathrm{F} 1$ ce qui est en accord avec l'hypothèse de l'origine génétique du manque de fertilité constaté.

On peut estimer l'effet des parents sur le taux de nouaison de leur descendance $\mathrm{F} 1$ et $\mathrm{F} 2$ en observant leur comportement avec un partenaire commun (tabl. 3). Ainsi les F1 avec « Nainari 60S» (U) permettent de classer «VPM » (V) comme le parent induisant le plus faible taux de nouaison mesuré sur l'épi entier, puis vient Maris Freeman (T) et enfin (Champlein $\times$ Aronde 68) (C) qui pour sa part donne la descendance la plus fertile. Par contre, on ne peut pas classer les partenaires de $\mathrm{C}$ utilisé comme parent récurrent ; les $F 1$ avec $U, V$ et $T$ ont toutes un taux de nouaison élevé. En F2 V et $\mathrm{T}$ se comportent de manière comparable avec $U$ alors que le taux de nouaison des $F 2$ CU et UC est plus élevé ; avec C la F2 CT montre un taux de nouaison plus faible que les autres croisements.

Seuls les rétrocroisements faisant intervenir $\mathrm{V}$ ont été rapportés dans le tableau 3. Lorsque $V$ est impliqué à 2 reprises dans les croisements, UVV et CVV, on constate une fertilité de l'épi entier moins bonne que dans les rétrocroisements où il n'intervient qu'une seule fois VUU, UVU et CVC. On peut remarquer à nouveau que le croisement UVU qui possède le cytoplasme $U$ est plus fertile que le croisement VUU avec le cytoplasme $\mathrm{V}$.

\section{B. Etude cytogénétique}

\section{Etude des variétés parentales}

Les lignées $U, V$ et $C$, cultivées en serre, présentent une majorité de plantes à $2 \mathrm{n}=42$ chromosomes ; mais la variété «Maris Freeman » $(\mathrm{T})$, dans l'échantillon observé, présente un pourcentage important de plantes à l'état aneuploïde, 6 plantes sur 28 ont $2 \mathrm{n}=41 \mathrm{chro}$ mosomes, 1 plante est double monosomique $(2 \mathrm{n}=40)$ et 1 est monotélosomique $(2 \mathrm{n}=41+\mathrm{t})$. Ces plantes aneuploïdes ne seront pas utilisées comme parents dans les croisements. Sur les 17 plantes observées de la lignée «VPM » (V) issue d'hybridations interspécifiques, une est monotélosomique. Les plantes parentales analysées pour leur fertilité et utilisées dans les croisements sont à $2 \mathrm{n}=42$ chromosomes et elles ont un comportement méiotique régulier (tabl. 4) : le nombre moyen d'univalents et de multivalents par cellule est faible et le pourcentage de cellules avec un trivalent ou un quadrivalent réduit.

\section{Etude des plantes FI en 1975 et 1976}

Chez les hybrides F1 observés en 1975 et 1976, les croisements réciproques ont un comportement méiotique identique. Pour chaque combinaison hybride, les données cytologiques des croisements réciproques ont donc été regroupées (tabl. 4). Le nombre moyen d'univalents des plantes F1 est plus élevé que celui des parents, notamment en 1976. Le nombre moyen de quadrivalents est aussi très important particulièrement lorsque les lignées $\mathrm{V}$ et $\mathrm{T}$ sont impliquées dans les croisements. En 1976, ces hybrides présentent en moyenne plus d'un multivalent par cellule.

Les différences de fréquence des associations chromosomiques observées en 1975 et en 1976 peuvent

TABLEAU 3

Taux de nouaison des $F 1, F 2$ et rétrocroisements.

Seed set in F1, F2 and back crosses.

\begin{tabular}{|c|c|c|c|c|}
\hline \multirow{2}{*}{$\begin{array}{l}\text { Nature génétique } \\
\text { du matériel végétal }\end{array}$} & \multicolumn{2}{|c|}{ Epi entier } & \multicolumn{2}{|c|}{4 épillets de la base } \\
\hline & Moyenne & Ecart-type & Moyenne & Ecart-type \\
\hline UV $F_{1}$ & 0,69 & 0,11 & 0,71 & 0,23 \\
\hline$F_{2}$ & 0,64 & 0,21 & 0,73 & 0,30 \\
\hline $\mathrm{VU} \mathrm{F}_{1}$ & 0,64 & 0,11 & 0,79 & 0,15 \\
\hline $\mathrm{F}_{2}$ & 0,57 & 0,27 & 0,65 & 0,37 \\
\hline UT $F_{1}^{2}$ & 0,78 & 0,18 & 0,81 & 0,19 \\
\hline $\mathrm{F}_{2}$ & 0,65 & 0,22 & 0,68 & 0,26 \\
\hline TU $F_{1}^{2}$ & 0,74 & 0,22 & 0,74 & 0,26 \\
\hline & 0,65 & 0,25 & 0,63 & 0,29 \\
\hline $\mathrm{UC} \mathrm{F}_{1}$ & 0,88 & 0,15 & 0,89 & 0,21 \\
\hline $\mathrm{F}_{2}$ & 0,78 & 0,26 & 0,76 & 0,32 \\
\hline $\mathrm{CU} \mathrm{F}_{1}$ & 0,88 & 0,10 & 0,92 & 0,13 \\
\hline$F_{2}$ & 0,74 & 0,26 & 0,70 & 0,29 \\
\hline $\mathrm{CV} \mathrm{F}$ & 0,75 & 0,10 & 0,86 & 0,16 \\
\hline $\mathrm{F}_{2}$ & 0,74 & 0,14 & 0,85 & 0,18 \\
\hline $\mathrm{CT} \mathrm{F}_{1}$ & 0,78 & 0,14 & 0,91 & 0,10 \\
\hline $\mathrm{F}_{2}$ & 0,65 & 0,22 & 0,76 & 0,28 \\
\hline U.V.U. & 0,78 & 0,12 & 0,81 & 0,20 \\
\hline V.U.U. & 0,70 & 0,20 & 0,69 & 0,29 \\
\hline U.V.V. & 0,56 & 0,20 & 0,68 & 0,21 \\
\hline C.V.C. & 0,65 & 0,21 & 0,62 & 0,31 \\
\hline C.V.V. & 0,58 & 0,18 & 0,68 & 0,33 \\
\hline Moyenne des F1 & 0,77 & 0,15 & 0,83 & 0,18 \\
\hline Moyenne des F2 & 0,68 & 0,22 & 0,72 & 0,29 \\
\hline
\end{tabular}


TABLEAU 4

Comportement méiotique de 4 lignées de blé et de leurs hybrides observés en 1975 et 1976.

Meiotic behaviour of 4 wheat lines and their hybrids observed in 1975 and 1976.

\begin{tabular}{|c|c|c|c|c|c|c|c|c|c|c|}
\hline \multirow{3}{*}{ Lignées ou F1 } & \multirow{3}{*}{$\begin{array}{l}\text { Nombre } \\
\text { de } \\
\text { plantes }\end{array}$} & \multirow{3}{*}{$\begin{array}{l}\text { Nombre } \\
\text { de } \\
\text { cellules }\end{array}$} & \multirow{2}{*}{\multicolumn{5}{|c|}{$\begin{array}{l}\text { Nombre moyen de configurations } \\
\text { par cellule-mère du pollen }\end{array}$}} & \multicolumn{3}{|c|}{$\%$ de cellules avec } \\
\hline & & & & & & & & \multirow{2}{*}{$\begin{array}{c}1 \\
\text { multi. }\end{array}$} & \multirow{2}{*}{$\begin{array}{c}2 \\
\text { multi. }\end{array}$} & \multirow{2}{*}{$\begin{array}{c}3 \\
\text { multi. }\end{array}$} \\
\hline & & & $\mathbf{I}$ & II & III & IV & & & & \\
\hline \multicolumn{11}{|l|}{ PARENTS 1975 et 1976} \\
\hline $\mathrm{U}:$ Naïnari $60 \mathrm{~S}$ & 26 & 1010 & 0,08 & 20,92 & & 0,02 & & 2 & 0,1 & \\
\hline $\mathrm{T}:$ Maris Freeman & 22 & 1067 & 0,20 & 20,88 & & 0,01 & & 1 & & \\
\hline $\mathrm{V}:$ VPM 1.1.1.2.R4 & 16 & 725 & 0,14 & 20,91 & & 0,01 & & 1 & & \\
\hline $\mathrm{C}:$ Champlein $\times$ Aronde & 26 & 1078 & 0,06 & 20,93 & & 0,02 & & 2 & & \\
\hline \multicolumn{11}{|l|}{ F1 1975} \\
\hline$U V+V U$ & 14 & 554 & 0,75 & 18,58 & 0,12 & 0,89 & 0,03 & 61 & 20 & 1 \\
\hline $\mathrm{UC}+\mathrm{CU}$ & 11 & 439 & 0,34 & 19,84 & 0,04 & 0,44 & 0,02 & 41 & 4 & \\
\hline $\mathrm{UT}+\mathrm{TU}$ & 9 & 341 & 0,42 & 18,94 & 0,05 & 0,87 & 0,01 & 75 & 9 & \\
\hline $\mathrm{CV}$ & 3 & 136 & 0,35 & 19,04 & 0,07 & 0,84 & & 81 & 5 & \\
\hline CT & 5 & 212 & 0,16 & 19,12 & 0,04 & 0,87 & & 76 & 8 & \\
\hline \multicolumn{11}{|l|}{ F1 1976} \\
\hline$U V+V U$ & 7 & 209 & 0,96 & 18,40 & 0,17 & 0,92 & 0,01 & 58 & 24 & 1 \\
\hline $\mathrm{UC}+\mathrm{CU}$ & 7 & 252 & 0,49 & 19,83 & 0,06 & 0,38 & 0,03 & 41 & 3 & \\
\hline $\mathrm{UT}+\mathrm{TU}$ & 8 & 344 & 1,00 & 18,24 & 0,16 & 1,01 & & 77 & 20 & \\
\hline $\mathrm{CV}$ & 5 & 245 & 0,51 & 18,51 & 0,07 & 1,05 & 0,01 & 78 & 18 & \\
\hline CT & 4 & 230 & 0,27 & 18,72 & 0,07 & 1,02 & & 90 & 10 & \\
\hline
\end{tabular}

$\mathrm{I}=$ univalent

II = bivalent

III $=$ trivalent

IV = quadrivalent autres multivalents $=$ penta, hexa, hepta... valent

1 multi $\quad=1 \mathrm{III} \quad \mathrm{IV}$

2 multi $\quad=2$ III ou 2 IV ou 1 autre multivalent

3 multi $\quad=3$ III ou 3 IV ou 1 autre multivalent +1 III ou IV s'expliquer par les conditions de culture des plantes. Les premières analyses ont été réalisées à partir de matériel cultivé en serre, tandis que les secondes ont été faites à partir de plantes cultivées en châssis froids à l'extérieur.

La structure chromosomique de V par rapport à certains blés est bien connue (DOUSSINAULT et al., 1974 ; BOURGEOIS et al., 1978) et elle est différente de celle de la lignée $\mathrm{U}$ à laquelle cette lignée a été confrontée. Le tableau 4 montre que 2 translocations réciproques différencient les lignées mises en présence. Cependant, dans le cas de croisements entre $\mathrm{C}$ et $\mathrm{U}$, le pourcentage de cellules avec 2 multivalents est faible. La fréquence de cellules avec un multivalent chez les 2 parents $U$ et $C$ est comparable à celle avec 2 multivalents chez les hybrides. On peut donc seulement dire qu'au moins une translocation réciproque différencie les 2 lignées. II faut noter également que les pourcentages de cellules avec multivalents sont similaires en 1975 et 1976 pour les hybrides réalisés avec U. Les quelques différences entre les 2 années observées au niveau des croisements $\mathrm{CV}$ et $\mathrm{CT}$ traduisent vraisemblablement une fluctuation due à un échantillonnage trop restreint.

\section{Analyse des plantes de la génération $F 2$ des croise- ments $U V$ et $V U$ en 1976}

L'analyse cytologique de quelques plantes F2 au niveau des croisements réciproques entre «VPM » (V) et « Nainari $60 \mathrm{~S}$ » $(\mathrm{U})$ révèle une hétérogénéité de comportement méiotique (tabl. 5). Certaines plantes F2 (UV 50, 59, VU 58) se comportent comme les parents alors qu'elles proviennent de plantes $F 1$ présentant un pourcentage important de cellules avec multivalents.
D'autres plantes $\mathrm{F} 2$ telles que UV 48, 49, 51 ou VU $41,52,72,94$ ont 10 p. 100, ou plus, de leurs chromosomes sous forme d'univalents et de multivalents. Ce type de matériel F2 apparaît intéressant pour estimer une éventuelle liaison entre les anomalies méiotiques et la stérilité.

\section{Fertilité pollinique chez les plantes $\mathbf{F} 2$ des croise- ments UV et VU. Relations avec le taux de nouai- son et le comportement méiotique}

Le taux de grains de pollen mal conformés et peu colorés au carmin ne varie pas de l'épiaison à l'anthèse sauf pour la plante F2 UV 45 qui passe de 3 à 20 p. 100. S'il est très faible pour les parents $(3 \mathrm{p}$. 100), il est souvent plus élevé en $\mathrm{F} 1$ et $\mathrm{F} 2$ et peut atteindre 35 p. 100 (VU 90). Certaines plantes F2 ont été analysées à la fois pour leur comportement méiotique en MI et leur fertilité pollinique estimée.

Le coefficient de corrélation entre le pourcentage de grains de pollen mal colorés à l'anthèse et le taux de nouaison de la même plante est de - 0,642, il est hautement significatif (fig. 1), une bonne fertilité pollinique est liée à une bonne fertilité en grain de l'épi (UV 76) et, a contrario, une mauvaise fertilité pollinique est liée à une mauvaise fertilité en grain de l'épi (UV 49 et VU 90). Pourtant, il semblerait que le nombre de grains de pollen normalement conformés et colorés par la technique de la coloration au carmin acétique soit suffisant dans tous les cas pour assurer la fécondation.

Pour expliquer ce phénomène, on peut faire l'hypothèse que les grains de pollen colorés provenant des étamines où le taux de grains de pollen anormaux est 
TABLEAU 5

Comportement méiotique de plantes F2 des croisements réciproques entre Nainari $60 \mathrm{~S}$ (U) et VPM.1.1.2.R4 (V) observé en 1976.

Meiotic behaviour of F2 plants from reciprocal crosses between Nainari $60 \mathrm{~S}$ (U) and VPM.1.1.1.2.R4 (V) observed in 1976.

\begin{tabular}{|c|c|c|c|c|c|c|c|c|c|c|}
\hline \multirow{3}{*}{$\mathrm{F} 2$} & \multirow{3}{*}{$\begin{array}{l}\text { Nombre } \\
\text { de } \\
\text { cellules }\end{array}$} & \multirow{2}{*}{\multicolumn{5}{|c|}{$\begin{array}{l}\text { Nombre moyen de configurations } \\
\text { par cellule-mère du pollen }\end{array}$}} & \multicolumn{4}{|c|}{$\%$ de chromosomes sous forme de } \\
\hline & & & & & & & & & & \\
\hline & & $\mathrm{I}$ & II & III & IV & autres & I & III & IV & multi-valents \\
\hline \multicolumn{11}{|l|}{ UV } \\
\hline pl 48 & 50 & 1,84 & 17,98 & 0,04 & 1,02 & & 4,4 & 0,3 & 9,7 & \\
\hline pl 49 & 50 & 0,46 & 17,72 & 0,14 & 1,30 & 0,08 & 1,1 & 1,0 & 12,4 & 1,1 \\
\hline pl 50 & 90 & 0,22 & 20,84 & & 0,07 & & 0,1 & & 0,6 & \\
\hline $\mathrm{pl} 51$ & 20 & 2,95 & 17,20 & 0,15 & 1,05 & & 7,0 & 1,1 & 10,0 & \\
\hline pl 59 & 60 & 0,20 & 20,80 & & 0,05 & & 0,5 & & 0,5 & \\
\hline $\mathrm{pl} 61$ & 50 & 0,08 & 20,36 & & 0,30 & & 0,2 & & 2,9 & \\
\hline pl 70 & 50 & 0,32 & 20,52 & & 0,16 & & 0,8 & & 1,5 & \\
\hline pl 76 & 50 & 0,20 & 20,64 & 0,04 & 0,10 & & 0,5 & 0,3 & 1,0 & \\
\hline pl 91 & 50 & 0,08 & 19,91 & 0,02 & 0,51 & & 0,2 & 0,2 & 4,9 & \\
\hline \multicolumn{11}{|l|}{$\mathbf{V U}$} \\
\hline pl 41 & 20 & 0,80 & 18,50 & & 1,05 & & 1,9 & & 10,0 & \\
\hline pl 52 & 50 & 1,22 & 18,82 & 0,14 & 0,68 & & 2,9 & 1,0 & 6,5 & \\
\hline $\mathrm{pl} 57$ & 50 & 0,64 & 20,64 & & 0,02 & & 1,5 & & 0,2 & \\
\hline pl 58 & 50 & 0,24 & 20,84 & & 0,02 & & 0,6 & & 0,2 & \\
\hline pl 72 & 30 & 0,40 & 18,77 & 0,06 & 0,97 & & 1,0 & 0,5 & 9,2 & \\
\hline pl 90 & 30 & 0,53 & 19,83 & 0,07 & 0,40 & & 1,3 & 0,5 & 3,8 & \\
\hline pl 94 & 39 & 0,27 & 18,95 & 0,05 & 0,92 & & 0,6 & 0,4 & 8,8 & \\
\hline
\end{tabular}

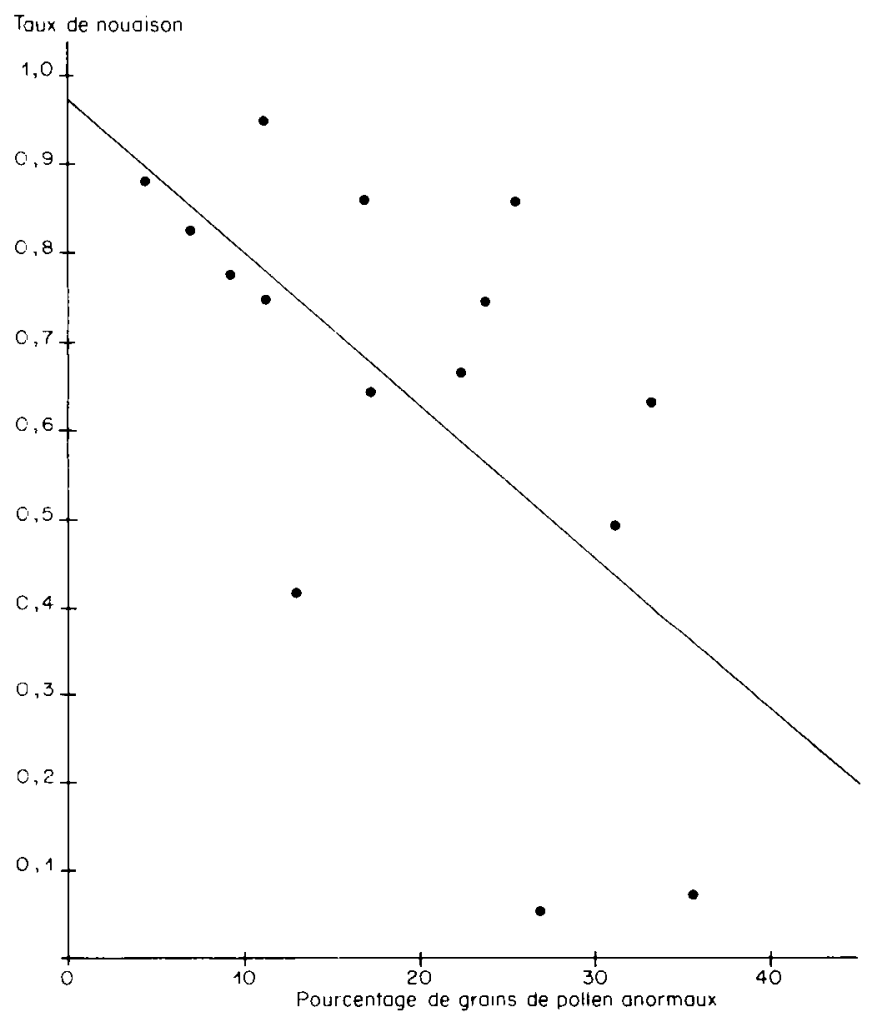

Figure 1

Relation entre le taux de nouaison et la qualité des grains de pollen à l'anthèse.

Relationship between level of seed set and pollen quality at anthesis.

élevé sont en fait subléthaux et incapables d'assurer la fécondation. Cette hypothèse est confortée par les résultats de HESLOP-HARRISON et al., 1984, qui indiquent que la technique de coloration au carmin surestime la viabilité réelle du pollen. On peut aussi avancer comme hypothèse complémentaire que la probabilité de réussite de la fécondation dépend de la quantité de pollen viable atteignant le stigmate ou que les fertilités mâles et femelles sont liées. C'est ce qu'observent SHARMA \& WAINES (1981) chez les blés diploïdes.

Le classement des plantes F2 des croisements UV et VU selon l'irrégularité de leur méiose estimée par le 
nombre moyen de chromosomes qui ne sont pas sous la forme de bivalents et selon leur taux de nouaison ne permet pas d'établir de corrélation entre les 2 observations: le coefficient de corrélation global est de - 0,332, il n'est pas significatif (fig. 2).

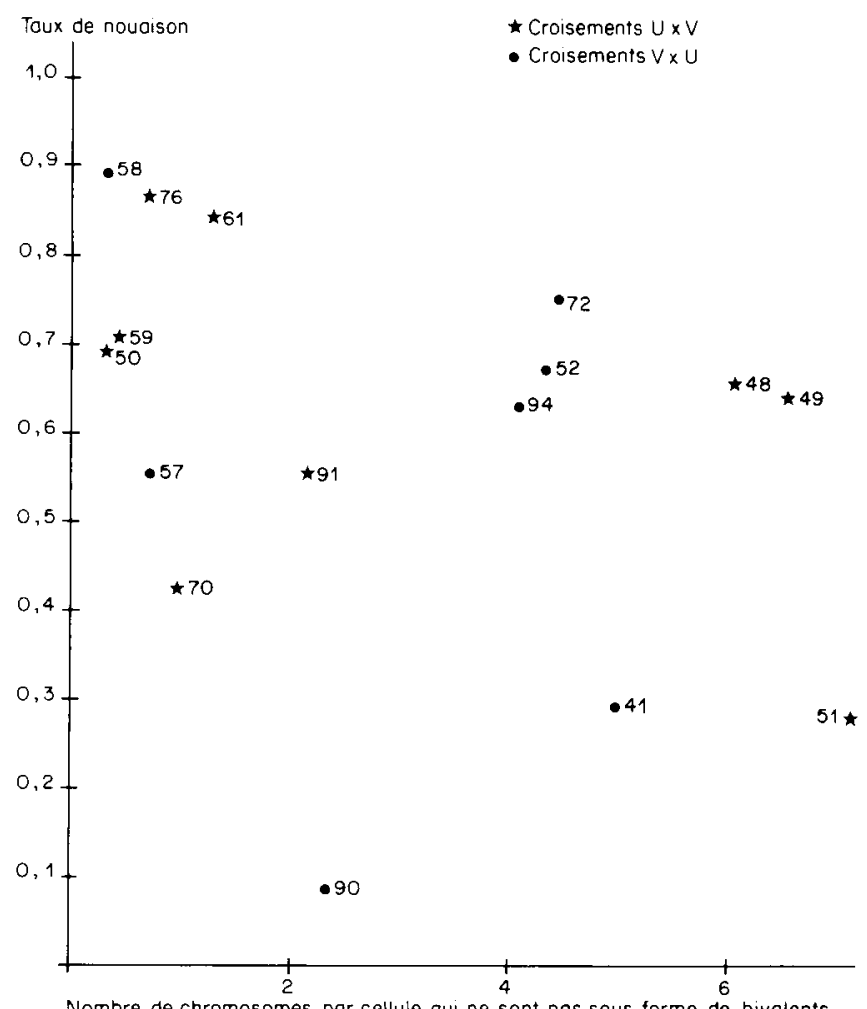

Figure 2

Relation entre le taux de nouaison et la régularité méiotique de plantes $F 2$ des croisements $U V$ et $V U$.

Relationship between level of seed set and the regularity of meiosis in +2 plants of crosses $U V$ and $V U$.

Cependant, on peut remarquer que seules les plantes ayant une méiose régulière ont un taux de nouaison supérieur à 0,8 . De même lorsque les anomalies méiotiques sont «faibles" (moins de 2 chromosomes par cellule qui ne sont pas sous forme de bivalents) le taux de nouaison des plantes correspondantes varie de 0,4 à 0,9 . Dans le cas d'anomalies plus importantes (plus de 2 chromosomes non appariés) le taux de nouaison varie de 0,1 à 0,75 .

L'étude simultanée du comportement méiotique, des grains de pollen et du taux de nouaison n'a été réalisée que sur 5 plantes mais il apparaît bien que les irrégularités méiotiques ne sont pas étroitement liées à un fort pourcentage de grains de pollen anormaux.

\section{CONCLUSION}

Les hypothèses concernant les causes du déficit de nouaison chez le blé tendre peuvent être nombreuses. Dans cet article, nous nous sommes intéressés à l'étude comparative de la régularité des méioses observées au stade métaphase I sur les cellules-mères des grains de pollen et du taux de nouaison.
Nous avons défini le taux de nouaison à partir du dénombrement des grains dans chacune des 2 premières fleurs des épillets comme critère de mesure du phénomène. En effet, le nombre moyen de grains par épillet intègre en plus l'aptitude génotypique à produire des grains.

Une corrélation négative nette a pu être mise en évidence entre le pourcentage de grains de pollen anormaux et le taux de nouaison sans qu'une explication physiologique ait été trouvée.

Il apparaît dans notre étude que certains génotypes « Maris Freeman » et surtout «VPM », ont des taux de nouaison plus faibles que les autres lignées. Ils donnent naissance à des Fl dont le taux de nouaison dépend du partenaire avec lequel ils sont combinés; le classement du taux de nouaison moyen des F2 est comparable à celui des $F 1$. Il n'est pas possible de mettre en évidence de lien net entre les irrégularités méiotiques observées, dues à la présence de translocations, et le taux de nouaison ; ainsi il semble bien que les différences de structures chromosomiques entre blés ne soient pas un obstacle majeur pour l'obtention de descendances fertiles de ces croisements. Cependant, le croisement CU présente la méiose la plus régulière et également le taux de fertilité le plus élevé. On ne retrouve pas l'influence négative de la lignée $U$ malgré la tendance à l'aneuploïdie constatée chez les génotypes demi-nains (WORLAND \& LAW, 1985).

L'étude plante à plante des F2 UV et VU ne montre pas de corrélation négative significative entre le taux de nouaison et le niveau de régularité de la méiose. Cependant, les plantes dont la méiose est très irrégulière ont un taux de nouaison faible. Cette conclusion rejoint celle de BANNIER (1978) à propos de croisements où est impliquée une translocation avec le seigle.

Cependant, certaines plantes qui présentent une méiose régulière ont, elles aussi, un faible taux de nouaison. On peut faire l'hypothèse de l'existence d'un système génétique qui contrôle le taux de nouaison lorsque les températures sont basses au moment des stades préméiotiques, ainsi, dans ces conditions, CHAPMAN \& MILLER (1981) ont localisé sur le chromosome $5 \mathrm{D}$ de Chinese Spring un gène affectant l'appariement chromosomique. En exerçant une pression de sélection, il devrait être possible d'éliminer les plantes partiellement stériles.

Il semble se superposer ici plusieurs phénomènes qu'il conviendrait d'analyser dans une étude ultérieure pour mieux comprendre ce phénomène et exercer une sélection plus efficace. En particulier, il faudrait avant tout expliquer avec précision les stérilités enregistrées par l'observation du développement des gamètes mâles, femelles et des embryons.

Il faudrait en déterminer les causes par l'action de températures contrôlées et par la caractérisation des stades critiques. BENNETT et al. (1972) montrent que c'est pendant l'interphase préméiotique que les températures basses ont un effet négatif sur les appariements chromosomiques sans toutefois relier cette observation à un défaut de fertilité. QUIAN et al. (1986) constatent pour leur part une diminution de la viabilité du pollen lorsque les températures basses interviennent au cours de sa maturation.

Nous avons évoqué la possibilité du contrôle génétique de la sensibilité des génotypes aux basses tempéra- 
tures. La mise au point de critères de sélection devrait permettre l'étude de la variabilité de l'espèce blé tendre pour ce caractère et la sélection de génotypes peu sensibles.

Reçu le 12 février 1987. Accepté le Ier février 1988.

\section{REMERCIEMENTS}

Les auteurs tiennent à remercier Madame Y. CAUDERON et Mademoiselle B. MAISONNEUve pour leur lecture attentive de l'article et leurs critiques constructives.

\section{RÉFÉRENCES BIBLIOGRAPHIQUES}

Bannier E., 1978. Investigations in fertility and its relationship with meiotic stability in monosomic F1 hybrids of wheat (Triticum aestivum L.). Arch. Zuchtungeforsch., 8 (6), 395-403.

Bennett M. D., Smith J. B., Kemble R., 1972. The effect of temperature on meiosis and pollen development in wheat and rye. Can. $J$. Genet. Cytol., 14 (3), 615-624.

Bourgeois F., Dosba F., Douaire G., 1978. Analyse et identification des translocations réciproques présentes chez les géniteurs VFM et les variétés « Marne », « Moisson » et « Roazon». Ann. Amelior. Plant., 28 (4), $411-429$.

Chapman V., Miller T. E., 1981. The location of a gene affecting meiotic chromosome pairing at low temperature in Triticum aestivum. Z. Pflanzenzücht., 86, 50-55.

Dosba F., Doussinault G., 1978. Création de lignées de blé présentant les caractéristiques agronomiques favorables d'Aegilops ventricosa. Ann. Amélior. Plant., 28 (1), 27-44.

Doussinault G., Koller J., Touvin H., Dosba F., 1974. Utilisation des géniteurs VPM I dans l'amélioration de l'état sanitaire du blé tendre. Ann. Amelior. Plant., 24 (3). 215-241.

Gale M. D., Marshall G. A., Rao M. V., 1981. A classification of the Norin 10 and Tom Thumb dwarfing genes in British, Mexican, Indian and other hexaploid bread wheat varieties. Euphytica, 30 (2), 355-361.

Heslop-Harrison J., Heslop-Harrison Y., Shivanna K. R., 1984. The evaluation of pollen quality and a further appraisal of the fluorochromatic FCR test procedure. TAG, 67, 367-375.
Maia N., 1967. Obtention de blés tendres résistants au piétin-verse (Cercosporella herpotrichoides) par croisements interspécifiques. $C$. R. Acad. Agric. Fr., 53 (2), 149-154.

Maisonneuve B., Philouze J., 1982. Action des basses températures nocturnes sur une collection variétale de tomate (Lycopersicon esculentum Mill.). II. Etude de la quantité et de la qualité du pollen. Agronomie, 2 (5), 453-458.

Quian C. M., Xu A., Liang G. H., 1986. Effects of low temperatures and genotypes on pollen development in wheat. Crop Sci., 26, 43-46.

Riley R., Coucoli H., Chapman V., 1967. Chromosomal interchanges and the phylogeny of wheat. Heredity, 22, 233-248.

Sharma H. C., Waines J. G., 1981. The relationships between male and female fertility and among taxa in diploid wheats. $A m$. J. Bot., 68 (3), 449-451.

Suneson C. A., 1962. Use of Pugsley sterile wheat in cross breeding. Crop Sci., 2, 534-535.

Watanabe Y., 1954. Studies on the cytological instabilities of common wheat. Jpn. J. Breed., 4. 67-77.

Worland A. J., Law C. N., 1985. Aneuploidy in semi dwarf wheal varieties. Euphytica, 34, 317-327. 